University of Texas at El Paso

ScholarWorks@UTEP

\title{
Geometric Approach to Detecting and Locating Cracks in Thin Plates by Lamb Wave Reflection: Case of Moving Transducer
}

Jose R. Mares

Roberto A. Osegueda

The University of Texas at El Paso, osegueda@utep.edu

Nagaswaroopa Kaukuri

Vladik Kreinovich

The University of Texas at El Paso, vladik@utep.edu

Follow this and additional works at: https://scholarworks.utep.edu/cs_techrep

Part of the Computer Engineering Commons

Comments:

UTEP-CS-04-05.

Published in: Tribikram Kundu (ed.), Health Monitoring and Smart Nondestructive Evaluation of Structural and Biological Systems III, Proceedings of the SPIE/International Society for Optical Engineering, Vol. 5394, San Diego, CA, March 14-18, 2004, pp. 385-398.

\section{Recommended Citation}

Mares, Jose R.; Osegueda, Roberto A.; Kaukuri, Nagaswaroopa; and Kreinovich, Vladik, "Geometric Approach to Detecting and Locating Cracks in Thin Plates by Lamb Wave Reflection: Case of Moving Transducer" (2004). Departmental Technical Reports (CS). 289.

https://scholarworks.utep.edu/cs_techrep/289

This Article is brought to you for free and open access by the Computer Science at ScholarWorks@UTEP. It has been accepted for inclusion in Departmental Technical Reports (CS) by an authorized administrator of ScholarWorks@UTEP. For more information, please contact Iweber@utep.edu. 


\title{
Geometric approach to detecting and locating cracks in thin plates by Lamb wave reflection: case of moving transducer
}

\author{
Jose Rodrigo Mares, Roberto Osegueda, Nagaswaroopa Kaukuri, and Vladik Kreinovich \\ FAST Center for Structural Integrity of Aerospace Systems \\ 500 West University Av, El Paso, TX, USA 79968 \\ The University of Texas at El Paso \\ El Paso, Texas 79968 \\ Contact E-mail Address: osegueda@utep.edu
}

\begin{abstract}
This paper portrays work for the development of a Lamb wave scanning method for the detection of defects in thin plates. The approach requires the generation of an ultrasonic $A_{o}$ and $S_{0}$-Mode Lamb Wave using an incident transmitter excited with a tone burst centered at a near non-dispersive frequency. With a fixed relative separation both transmitter and receiving transducer, remotely scan a specific line section of the plate. The arrival time information coming from incident and reflected waves contain information associated with the location of reflection surfaces or potential flaws. The Hilbert-Huang transform is applied to the intrinsic mode functions which permit the computation of the signal energy as a function of time, proportional to the square of the amplitude of the analytical signal. The arrival times and amplitudes of the notch-reflected energy are used to calculate by means of two geometric methods, the coordinates of the source of the reflections. The resulting coordinates outline the extent and relative direction of notches in two different scenarios. One is having notches in a 0 to 22.5 degree orientation in respect to the far edge of the plate and two with notches of various sizes at a single rivet hole. Results of experiments conducted on 1.6mm-thick Aluminum square plates, with an arrangement of notches as described compare favorably with the actual notches.
\end{abstract}

Key words: Lamb waves, ultrasonic, waveforms, nondestructive evaluation, defect localization.

\section{INTRODUCTION}

Material deterioration is expected within the design life of structures and mechanisms, such as buildings, heavy machinery, pipes, offshore structures and aircrafts. Critical loading and temperature conditions are common conditions that eventually lead to some degree of deterioration. This deterioration can manifest in forms of internal cracking, flaws, corrosion in metallic parts and other forms of damage. Visual inspection has failed to localize and analyze theses forms of damage, especially when they are located in hidden regions. The need for rapid and reliable maintenance procedures and nondestructive techniques (NDE) that can be applied to large areas of the structure is a wide-ranging concern in today's industry. The use of Lamb waves is a new approach that has potential for improving large area inspections.

One of the advantages of ultrasonic testing by use of Lamb waves is the capability of long range inspections as previously recognized by Cawley and Alleyne et al. [1]. They pointed out that the key for the successful application of the method is the proper excitation of a single mode at a frequency located in a near non-dispersive region. Kundu et al. [2] introduced a scanning technique for composite matter using leaky Lamb waves using a pitch catch mode involving and immersed transmitter and a receiver. Their technique was effective in detecting internal defects such as missing fibers and fiber breakage. They examined the effectiveness of several modes and found that the $A_{o}$ mode was the most effective for the localization of the flaws. Kim and Song et al [3] studied the relationship between incident angle and phase velocity by demonstrating that a given frequency, incidence angle, and plate thickness, several modes may propagate with different velocities Lowe et al. [4] studied issues on Lamb wave sensitivity to defects and optimum selection of modes as related to defect size and strength of wave reflections. 
Other studies have focused on sensitivity issues to detect certain forms of damage. Ghosh et al. [5] studied Lamb wave propagation in large plates and its use in internal defect detection by computing stress fields inside the plate for different lamb modes. Kundu et al [2] investigated the effectiveness of using Lamb waves for the detection of kissing bonds, defined as closed cracks under compressive normal stresses. Their technique was based on the proper selection of the A1 mode, which proved sensitive to detection using ultrasonic guided waves. Chang and Mal [6] theoretically and experimentally investigated Lamb waves propagating in an infinite plate containing a circular hole with or without edge cracks. The theoretical analysis was accomplished by means of a hybrid method involving finite elements with special boundary condition fields representing Lamb wave modes. Measured time histories and amplitude spectra of the transmitted and reflected waves compared favorably with those calculated from the hybrid model. Chang and Mal [6] also observed that when Lamb waves pass through a region with cracks or material loss, some energy is reflected back due to back scattering and the transmitted waves are modified due to forward scattering. The presence of material loss, cracks or other flaws in the path of the incident Lamb waves can be detected and, in principle, characterized by analyzing the characteristics of the reflected and transmitted waves.

This paper deals with the detection of notches using a line scan approach of ultrasonic Lamb waves propagating in the $A_{o}$ and $S_{o}$ mode in thin plates with constant thickness. The approach relies on the generation of narrow-band pulse waves with a center frequency in the non-dispersive region of the Lamb-Wave dispersion curve. In this scanning approach transmitter and receiver where housed within a Plexiglas bubbler and mounted in a scanning arm where they moved simultaneously, the incident angle of the transmitter was set to 14 degrees for excitation of the $S_{o}$ mode and 27 degrees for the $\mathrm{A}_{\mathrm{o}}$ mode. The signals acquired from a wide-band ultrasonic receiver set an angle of 14 and 27 degrees respectively from the vertical axis, are processed using the Hilbert - Huang transform to determine wave energy as a function of time. The points of maximum energy are used to determine arrival times. From the arrival times acquired at two different receiver positions, the distances traveled form the source to the flaw, and to the receiver serve as the bases for geometrical solutions that will render the flaw location and orientation in the two scenarios discussed. For the rivet hole-notch combination an additional analysis is performed, which will identify the region not affected by the wave back scattering due to the rivet hole geometry.

\section{EXPERIMENTAL SETUP}

A usual specimen submitted to testing in this investigation is illustrated in Figure 1. The specimens consisted of $6061-$ T6 610mm by $910 \mathrm{~mm}$ (24 by $36 \mathrm{in}$.) rectangular Aluminum plates with a thickness of $1.60 \mathrm{~mm}(1 / 16 \mathrm{in}$.) and a $1.27-\mathrm{cm}$ (0.5 in.) center hole. Various plates were fabricated with two types of notches for those with angle and horizontal notches a $0.8-\mathrm{mm}$ depth was set, with lengths of $89 \mathrm{~mm}$ (3.5 in.) and $100 \mathrm{~mm}$ (4 in.). The other type involved full thickness notches ranging form $3.2 \mathrm{~mm}$ (1/8 in.) to $25.4 \mathrm{~mm}$ (1 in.) long in $3.2 \mathrm{~mm}$ increments, this notches were fabricated in combination to a $1.27-\mathrm{cm}(0.5 \mathrm{in}$.) center hole. Sending a signal from a function generator through a continuous amplifier to a transmitter enclosed by a bubbler created the ultrasonic Lamb waves. The wedge incidence angle was set at two values 14 degrees and 29 degrees in order to excite the $S_{o}$ and $A_{o}$ mode respectively. The receiver was a wide-frequency type housed within a bubbler. The transmitter-receiver arrangement was mounted in a scanning arm for simultaneous movement. Transmitter and receiver housing make possible to maintain uniform coupling with the plate. The design of both components facilitated the signal analysis, by providing sufficient distance between plate and transducer a clear travel time gate will develop between reflections from potential defects. The receiver signals were sent to a data acquisition system through a pre-amplifier. By means of a siphon mechanism the transmitter-receiver chambers are filled with water, which in turn create a thin film of water between the plate and each transducer, any excess water is drawn by two small vacuum pumps. The scanning table and the acquisition system are controlled using NDE acquisition software. The signals collected from the receiver as a function of its position were stored for further analysis. Figure 2 illustrates plate specimens on the scanner system. 
Aluminum Plate (6061-T6)

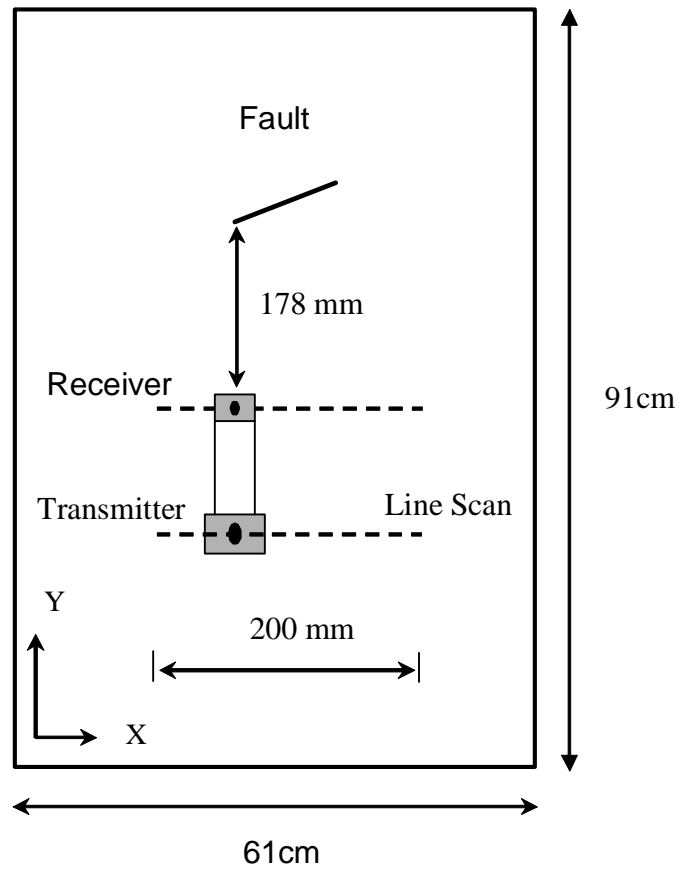

(a)

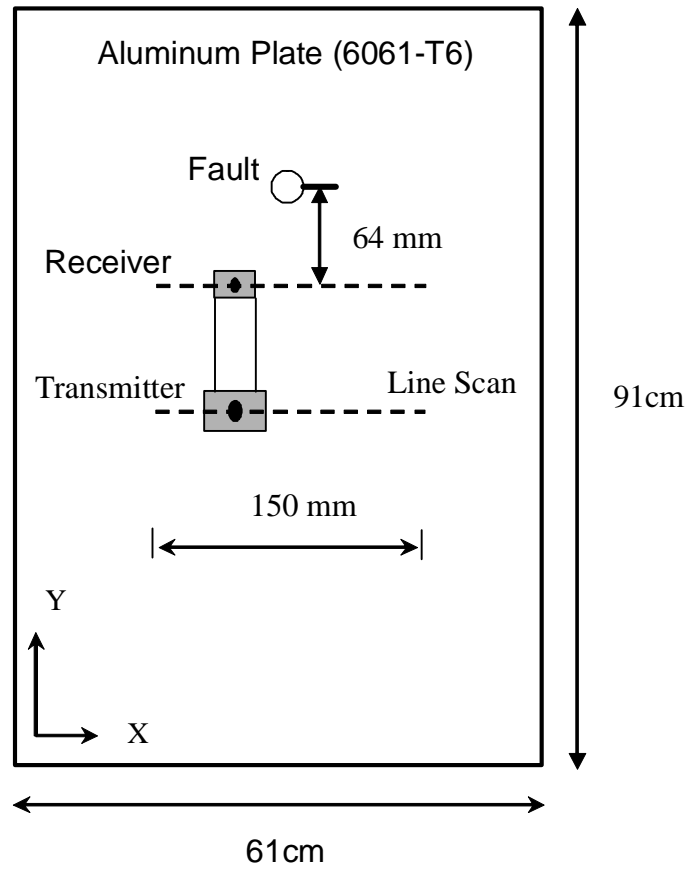

(b)

Figure 1. Schematic of line-scan approach to characterize notches. (a) Schematic of Aluminum plate specimens with notches. (b) Schematic of Aluminum plate specimens with rivet hole and notch.
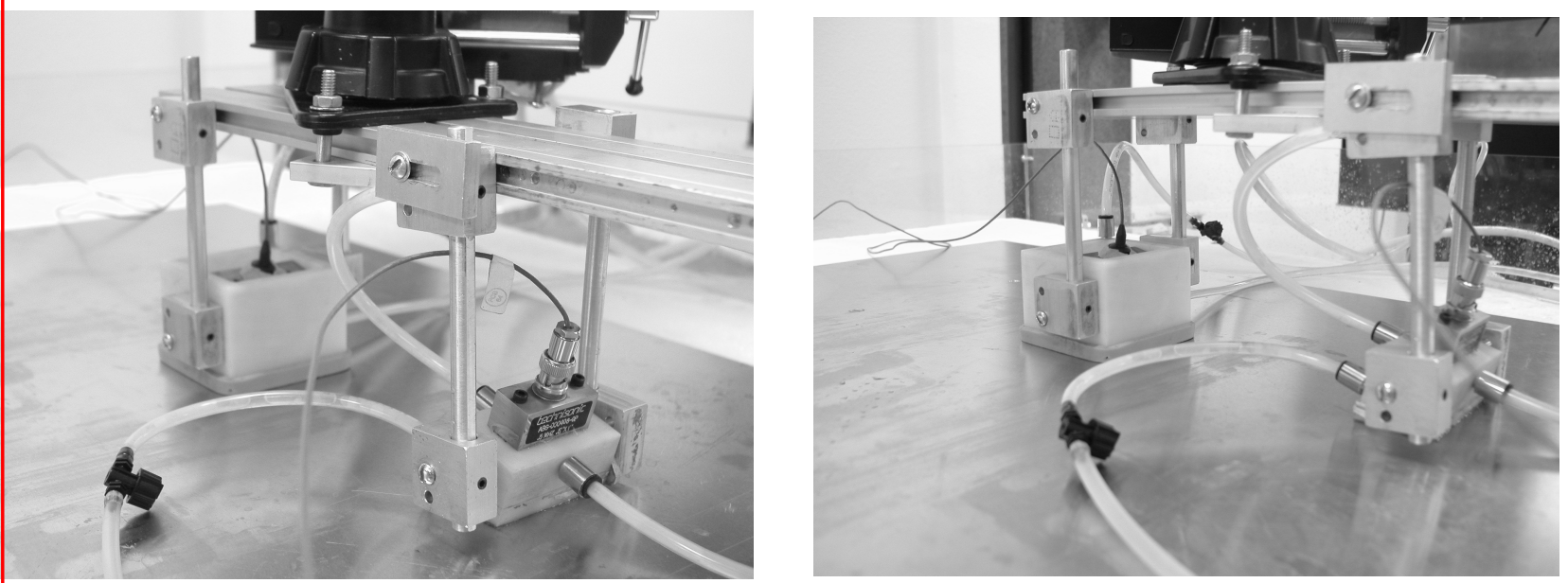

Figure 2. Scanning system with transmitter and receiver bubbler attached to scanning arm. 
In order to select the proper choice of frequency to attain near non-dispersive conditions, experimental phase dispersion curves were obtained and compared to the classical closed form solution originally published by Viktorov [7]. The experimental curves were obtained from data acquired by sending a rectangular pulse to the transmitter while data is collected along a line perpendicular to the transmitter at equally spaced intervals. The double FFT of the data array provides three-dimensional information of the propagating wave amplitudes versus frequency and wave number. Through the use of standard expressions, the graph is transformed to show the propagating wave amplitudes versus frequencies and phase velocity to obtain the experimental phase dispersion curve. This curve is illustrated in Figure 3 along with Viktorov's [7] analytical solution using the geometric and materials properties corresponding to the plate specimens. A good correlation was obtained. This experiment was also helpful to evaluate the frequency limitations of the transmitter and the receiver system. Reasonably good information was obtained for frequencies less than $2 \mathrm{MHz}$. In order to avoid different group and phase velocities, further tests were conducted by sending a $500-\mathrm{kHz}$ tone burst. The $S_{o}$ and $A_{o}$ mode propagates at a phase velocity of $5,280 \mathrm{~m} / \mathrm{s}$ and $2,300 \mathrm{~m} / \mathrm{s}$ respectively at this frequency.

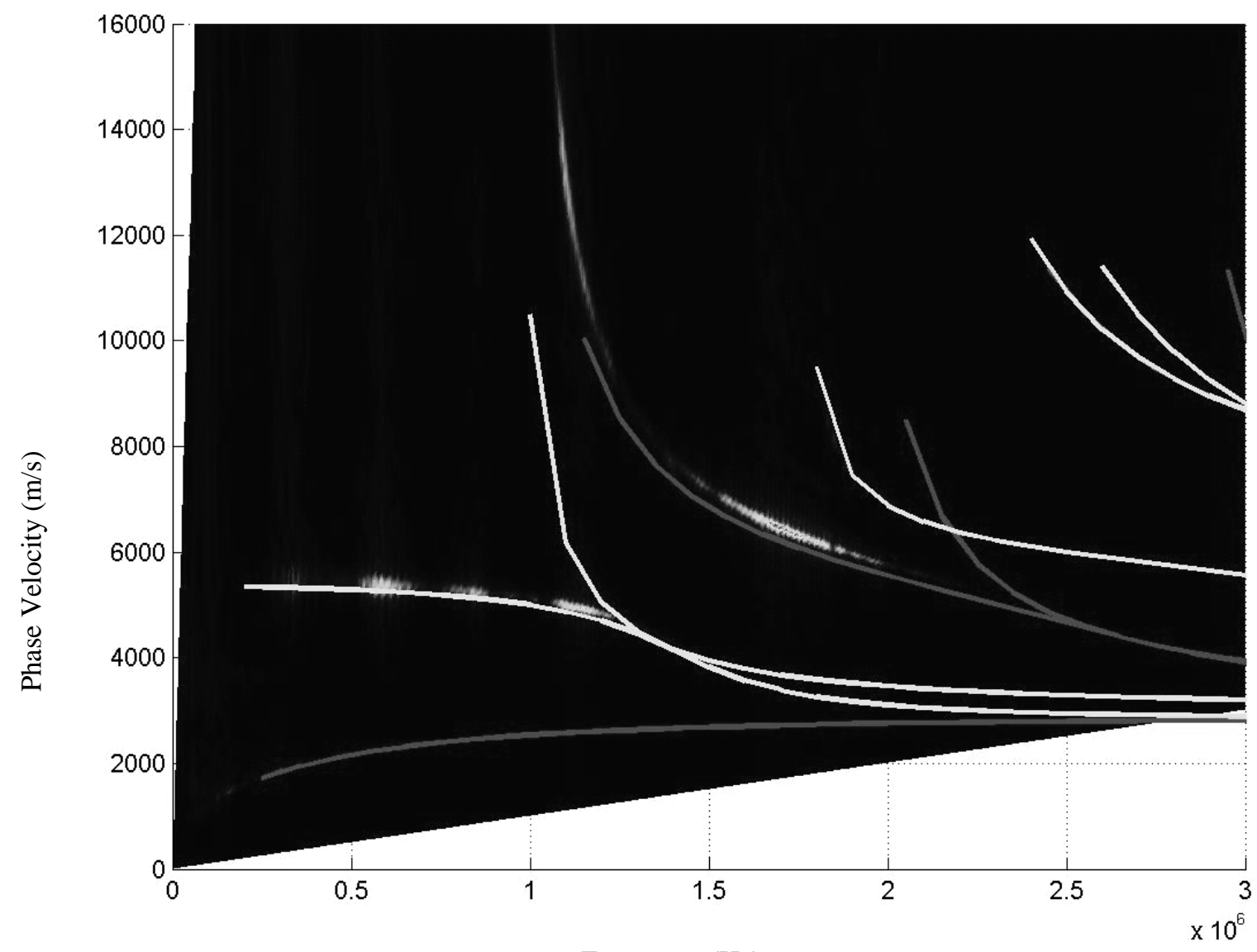

Frequency $(\mathrm{Hz})$

Figure 3. Experimental phase dispersion contour plots vs. analytical solution for Aluminum plate $1.6 \mathrm{~mm}$ thick.

The scanning strategy selected was based in the visualization of Lamb wave scattering and reflections from notch and hole combination conducted by Osegueda et al. [8], and DeVilla et al. [9]; these animations served as a visualization tool. 


\section{DETERMINATION OF SIGNAL ENERGY}

The Hilbert-Huang Transform is a recent breakthrough in the analysis of nonlinear no stationary time series analysis [10] which allows for the separation of the time series into intrinsic mode functions and a residue. The separation consists of a sifting process obtained by detecting the maximum and minimum peaks of the time series, computing of the maximum and minimum envelopes through a curve fitting approach, and subtracting the signal from the mean of the envelopes. For further details of the sifting process and the determination of the intrinsic mode functions see reference [10]. At the end, the intrinsic mode functions have the properties that the numbers of extrema and zero crossings must be equal or differ by one, and that at any point in time, the mean value of the envelopes defined by the local maxima and local minima is zero [10]. Figure 5 illustrates the results of the sifting process applied to a typical ultrasonic signal acquired by the receiver. The first graph is the original signal. The rest of the time series are the intrinsic mode functions, $C_{k}$, as obtained by applying the algorithms described in [10]. The last time series corresponds to the residue. When the $C_{k}$ 's are added, the original signal is recovered. It should be noted that the effect of the sifting, in context to Lamb waves, is filtering the data in a band-pass form. In essence, frequency ranges, whose wave groups may travel at different velocities, separate the time series. For example, the experiment phase dispersion curves associated with the first intrinsic functions of an array of time series acquired by sending a $500-\mathrm{kHz}$ tone burst to the transmitter and collecting data at equally spaced intervals along a line in the direction of the wave propagation is illustrated in Figure 6. Noise and the coincidental generation of waves at different frequencies can be controlled limiting the number of intrinsic mode functions.

\section{LINE SCANNING APPROACH}

In order to implement a solution for the angle notch, horizontal notch, and notch characteristics at the rivet hole, receiving signals are required along a line that can pick notch-reflected wave energy. Knowing the arrival times of notch-reflected waves, it is possible to use the information of two or more receiver locations to calculate the location of points on the surface that cause those reflections. However, in the case dealing with notch at a rivet hole the data measured along a line additionally picks up the scattered waves from the hole geometry. These would need to be separated. This paper considers a scanning approach along a line that is parallel to the notch orientation, as depicted in Figure 1, where the signals from the receiver at the known position can be processed to determine arrival times and coordinates of the source of reflections, providing information about the notch geometry. With the transmitter and receiver attached to the scanning arm, and align as illustrated in Figure1, so that the ultrasonic pulse is directed towards the receiver. The scanned line was parallel to the edge and placed so that the ultrasonic pulse is reflected from the notch would reflect towards the receiver. The scanned line was parallel to the edge at a distance from the fault of $178 \mathrm{~mm}$ from the notch in the case of angle and horizontal notches, and $64 \mathrm{~mm}$ for the rivet-notch combination. A total length of $200 \mathrm{~mm}$ and $150 \mathrm{~mm}$ was scanned for each respective scenario, and measurements were taken at $2 \mathrm{~mm}$ intervals. Such a scanning approach has the potential to be implemented in real-time.

All the acquired time series were processed using the Hilbert-Huang transform and the information was converted to signal energy using equations (1) through (5) and using the first three intrinsic mode functions. Figure 3 a) illustrates the signal wave energy for a horizontal notch while b) illustrates the wave energy for the hole-notch arrangement, as a function of time for all points along the scanned line. The horizontal axis corresponds to the time interval number, and the y-axis corresponds to the artificial offsets to visualize all the points along the line. The figure illustrates bumps corresponding to wave energy at different times. The first major bump corresponds to the front wave arriving at the receiver locations. The second major bump corresponds to a wave energy combination of scattered waves from the hole and reflected energy for the notch. The rest of the waves correspond to secondary reflection form the edges. It should be noted that the trigger was synchronized with the pulse leaving the function generator. There is at time delay corresponding to $50 \mu \mathrm{s}$ (310 time steps) or the time it takes a wave to travel through the water medium into the Aluminum plate after the trigger, and from the plate through the water into the receiver. The peaks of the energy provide the information of the arrival times along the wave pathways, and since the receiver and the transmitter positions and the propagation velocity are known, distance along the pathways can be determined. Figure 4 illustrates the arrival times of energy peaks that are above a given threshold. Figure 5 illustrates the maximum amplitude of the wave energy scattered by the hole versus the receiver position, corresponding to a control plate wit the hole without a notch. These graphs illustrate that the arrival times due to hole back scattering occur from 40 to $95 \mathrm{~mm}$ along the scan line. Reflections due to the notches would occur outside this region. 


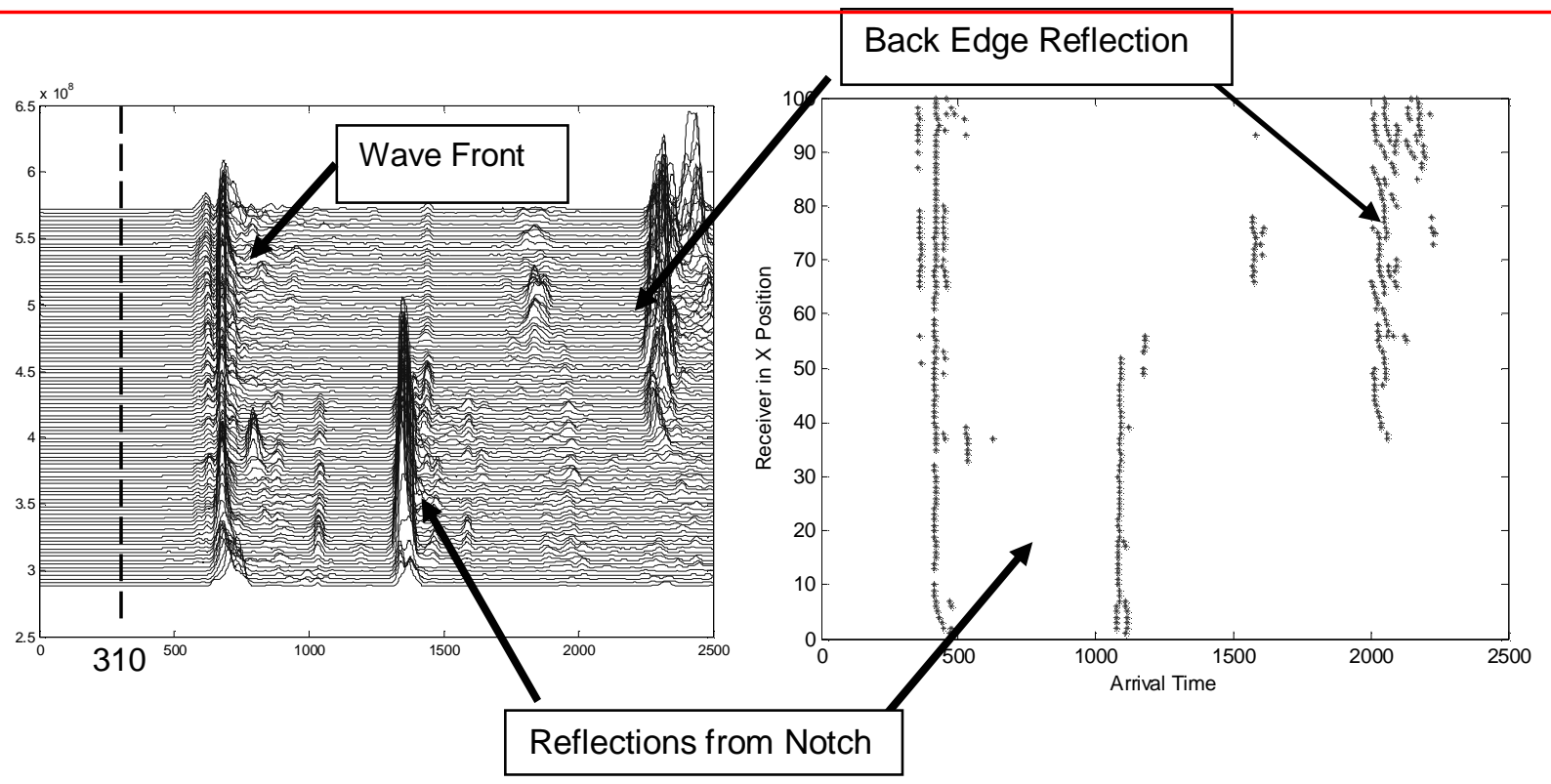

(a)

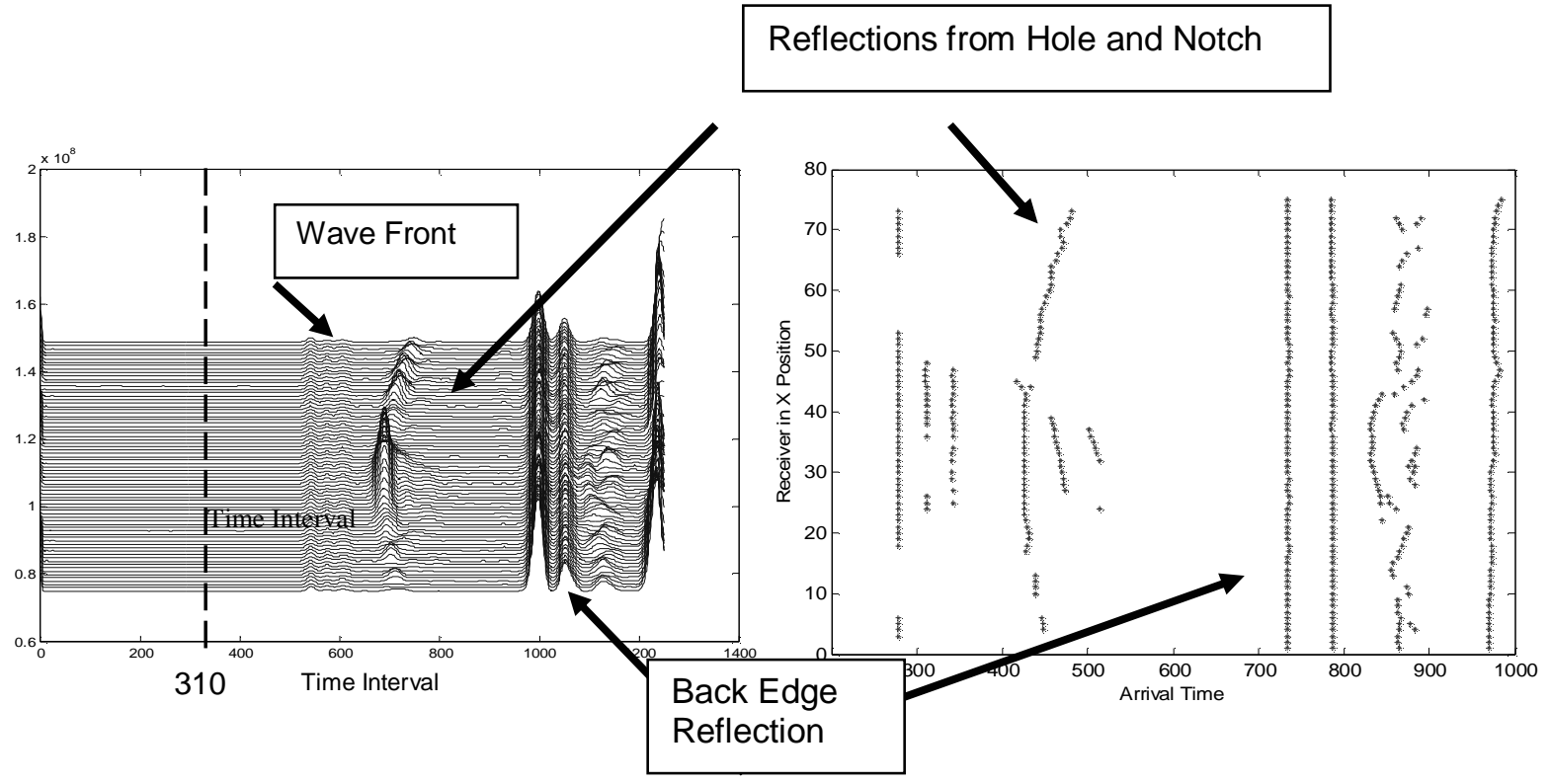

(b)

Figure 4. (a) Left: Wave energy display for horizontal notch along points of a typical scan. Right: Energy pulse arrival times. (b) Left: Wave energy display for hole-notch along points of a typical line scans. Right: Energy pulse arrival times. 


\section{SOLUTION OF NOTCH LOCATION}

Coordinates of points on a notch that causes reflection can be obtained using the times of flight of the incident and reflected waves from a fixed transmitter to a receiver at two different points. The approach is documented in reference [9]. However this approach required iterative methods to solve the resulting geometric problem, as well as decreasing the signal energy as the receiver moves away from the receiver. An alternative idea is to simultaneously move transmitter and receiver at a fixed distance, and to apply two geometrical methods (Geometric Method I and Geometric Method II).

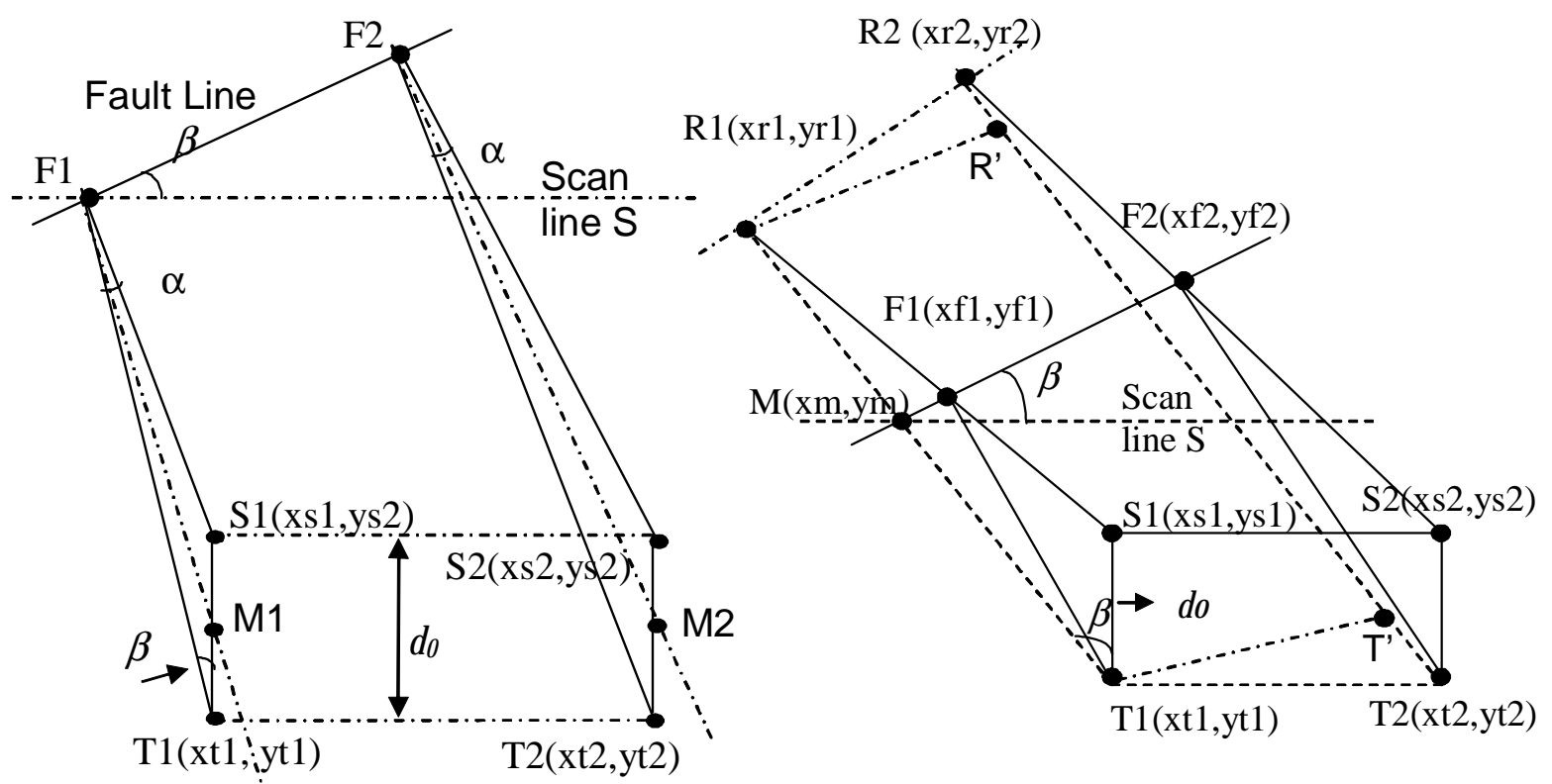

(a)

(b)

Figure 5. Geometric representations of reflected patterns. (a) Geometric method I. (b) Geometric Method II.

\section{Geometric Method I.}

The approach considered by the Geometric Method I, takes into account the simultaneous movement of transmitter T and sensor $\mathrm{S}$ along the scan line $\mathbf{s}$. The algorithm considers the transmitter and sensor to be fixed relative to one and other, along the $\mathrm{Y}$ - axis at a distance denoted by $d_{o}$, refer to Figure 1. The transmitter excites Lamb waves of a stable mode in the non-dispersive region, where waves propagate radially at a constant phase velocity. After performing energy analysis to obtain the arrival times of the incident wave and the reflected wave from a flaw $F_{1} F_{2}$, the distances $\mathrm{d}_{1}$ and $\mathrm{d}_{2}$ where calculated.

Depending on the orientation of the flaw in respect to the far edge of the plate, certain conditions can be expected if the reflection obtained is from the same flaw, distance $d_{1}$ and $d_{2}$ are constant for a horizontal flaw, as for flaws with different orientation, the angle $\beta$ between the line $\mathrm{F}_{1} \mathrm{~F}_{2}$ and $\mathbf{s}$ will remain the same see Figure 5 (a). 


$$
\begin{aligned}
& \text { The equations describing the distances and solution for the angle } \beta \text { are described as follows: } \\
& d_{i}={\overline{T_{i} F_{i}}}_{i}+\overline{F_{i} S_{i}} \\
& \overline{F_{i} M_{i}}=\frac{\bar{T}_{i} F_{i}+\bar{F}_{i} S_{i}}{2}=\frac{d_{i}}{2} \\
& \operatorname{Sin} \beta=\frac{d_{2}-d_{1}}{2 s} \\
& X_{i}=S_{i}-\overline{F_{i} M_{i}} \cdot \operatorname{Sin}(\beta) \quad(\mathrm{i}=1,2) \\
& Y_{i}=\left(S_{i}-\frac{d_{o}}{2}\right)+\overline{F_{i} M_{i}} \cdot \operatorname{Cos}(\beta) \quad(\mathrm{i}=1,2)
\end{aligned}
$$

\section{Geometric Method II}

For this method the data input, experimental setup, and nomenclature of terms reminded the same as for Geometric Method I. However this method involves the analysis of a trapezoid like shape constructed by the propagation and mirror reflections of the waves. In this method the distance corresponding to $d_{1}$ and $d_{2}$ will equal $d_{i}=T_{i} F_{i} S_{i}$ measured at a point $S_{i}$ along the scan line, this distance will also represent the distance $S_{i} R_{i}$. The point $R_{i}$ represents the mirror image of the path $\mathrm{T}_{\mathrm{i}} \mathrm{F}_{\mathrm{i}}$ assuming as the axis of symmetry the fault line $\mathrm{F}_{1} \mathrm{~F}_{2}$ see Figure 6 (b). We denote $\beta$ as the angle between the fault line $\mathrm{F}_{1} \mathrm{~F}_{2}$ and the direction of the scan line $\mathrm{S}_{\mathrm{i}}$ in which the receiver moves. Knowing that $\mathrm{T}_{\mathrm{i}} \mathrm{S}_{\mathrm{i}} \perp \mathrm{S}_{\mathrm{i}}$, and, due to the properties of reflection, $\mathrm{R}_{\mathrm{i}} \mathrm{T}_{\mathrm{i}} \perp \mathrm{F}_{\mathrm{i}}$; therefore, the angle $\angle \mathrm{R}_{\mathrm{i}} \mathrm{T}_{\mathrm{i}} \mathrm{S}_{\mathrm{i}}$ between $\mathrm{R}_{\mathrm{i}} \mathrm{T}_{\mathrm{i}}$ and $\mathrm{T}_{\mathrm{i}} \mathrm{S}_{\mathrm{i}}$ is also equal to $\beta$.

Due to the properties of reflection and when $i$ assumes values of 1 and 2 corresponding to any pair of points, the distance $R_{1} R_{2}$ is equal to $T_{1} T_{2}=s$. Since $T_{1} T_{2} \| S_{1} S_{2}$, the line $T_{1} T_{2}$ is at an angle $\beta$ to the reflection line $F_{1} F_{2}$, hence $R_{1} R_{2}$ is also at angle $\beta$ from the reflection line; see Fig. 4. If from $R_{1}$ and $T_{1}$ we draw the lines $R_{1} R^{\prime}$ and $T_{1} T^{\prime}$ that are parallel to $F_{1} F_{2}$ (and which are hence orthogonal to $R_{1} T_{1}$ and $R_{2} T_{2}$ ), then we conclude that $R_{1} R^{\prime}=T_{1} T^{\prime}=s \cdot \sin (\beta)$ hence:

$\mathrm{R}_{2} \mathrm{~T}_{2}-\mathrm{R}_{1} \mathrm{~T}_{1}=\mathrm{R}_{1} \mathrm{R}^{\prime}+\mathrm{T}_{1} \mathrm{~T}^{\prime}=2 s \cdot \sin (\beta)$

If we denote an average of $R_{1} T_{1}$ and $R_{2} T_{2}$ by $M$, we can thus conclude that $R_{1} T_{1}=M-s \cdot \sin (\beta)$ and $\mathrm{R}_{2} \mathrm{~T}_{2}=\mathrm{M}+s \cdot \sin (\beta)$. 


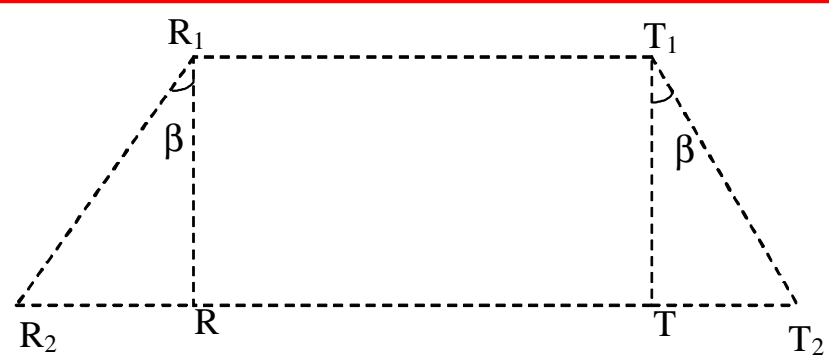

Figure 6. Schematic of developed geometry for Method II.

If the triangle $\Delta \mathrm{R}_{1} \mathrm{~T}_{1} \mathrm{~S}_{1}, \mathrm{R}_{1} \mathrm{~T}_{1}=\mathrm{M}-s \cdot \sin (\beta), \angle \mathrm{R}_{1} \mathrm{~T}_{1} \mathrm{~S}_{1}=\beta, \mathrm{T}_{1} \mathrm{~S}_{1}=d_{0}$, and $\mathrm{R}_{1} \mathrm{~S}_{1}=d_{1}$; therefore, the Law of Cosines leads to

$d_{2}{ }^{2}=(M-s \cdot \sin (\beta))^{2}+d_{o}{ }^{2}-2(M-s \cdot \sin (\beta)) \cdot d_{o} \cdot \cos (\beta)$

Similarly,

$d_{1}^{2}=(M+s \cdot \sin (\beta))^{2}+d_{o}^{2}-2(M+s \cdot \sin (\beta)) \cdot d_{o} \cdot \cos (\beta)$

Subtraction (3) from (4), we conclude that:

$d_{2}^{2}-d_{1}^{2}=4 M \cdot s \cdot \sin (\beta)-4 s \cdot d_{o} \sin (\beta) \cdot \cos (\beta)$

hence for

$Z_{1}{ }^{\text {def }}=\frac{\left(d_{2}^{2}-d_{1}^{2}\right)}{4 s}$

we get the formula:

$Z_{1}=\sin (\beta) \cdot\left(M-d_{o} \cos (\beta)\right), \quad Z_{1}{ }^{2}=\sin ^{2}(\beta) \cdot\left(M^{2}-2 M d_{o} \cos (\beta)+d_{o}{ }^{2} \cdot \cos ^{2}(\beta)\right)$

$Z_{2}{ }^{d e f}=\left(d_{2}^{2}+d_{1}^{2}\right) / 2=M^{2}+s^{2} \cdot \sin ^{2} \beta+d_{o}{ }^{2}-2 M \cdot d_{o} \cdot \cos (\beta)$

Once these formulas have been established a quadratic equation in terms of the unknown $\sin ^{2}(\beta)$ is introduced, along with expressions that will make possible a representation of the flaw points in Cartesian coordinates. Where $i=1,2$

$$
\begin{gathered}
Z_{2} \cdot \sin ^{2}(\beta)-Z_{1}{ }^{2}=\left(s^{2}+d_{o}{ }^{2}\right) \sin ^{4}(\beta) \\
\sin ^{2}(\beta)=\frac{-Z_{2}+\sqrt{Z_{2}{ }^{2}-4 Z_{1}{ }^{2} \cdot\left(s^{2}+d_{0}{ }^{2}\right)}}{2\left(s^{2}+d_{0}{ }^{2}\right)} \\
M=\frac{Z_{1}}{\sin (\beta)}+d_{0} \cdot \cos (\beta) \\
R_{1} T_{1}=M-s \cdot \sin (\beta) \quad R_{2} T_{2}=M+s \cdot \sin (\beta)
\end{gathered}
$$


Coordinates of Reflection points $\mathrm{R}_{1}, \mathrm{R}_{2}$
$\quad y_{R_{i}}=y_{T_{i}}+R_{i} T_{i} \cdot \cos (\beta)$
$\quad x_{R_{i}}=x_{T_{i}}-R_{i} T_{i} \cdot \sin (\beta)$
Coordinates of Mid points $\mathrm{M}_{1, \mathrm{M}_{2}}$
$\quad x_{M i}=\frac{x_{T_{i}}+x_{R_{i}}}{2} \quad y_{M i}=\frac{y_{T_{i}}+y_{R_{i}}}{2}$
Coordinates of the Fault line $\mathrm{F}_{1} \mathrm{~F}_{2}$
$y_{F i}=y_{M_{i}}+$ tan $(\beta) \cdot\left(x_{F i}-x_{M i}\right)$
$y_{F i}=y_{R_{i}}+\frac{y_{S_{i}}-y_{R_{i}}}{x_{S_{i}}-x_{R_{i}}}\left(x_{F_{i}}-x_{R_{i}}\right)$
Receiver and Transmitter Coordinates
Receiver Position X-axis $=\mathrm{X}_{\mathrm{S}_{\mathrm{i}}}=\mathrm{X}_{\mathrm{T}_{\mathrm{i}}}$
Receiver Position Y axis $=\mathrm{Y}_{\mathrm{S}_{\mathrm{i}}}=\mathrm{Y}_{\mathrm{T}_{\mathrm{i}}}$

\section{LOCALIZATION RESULTS}

The generation of $S_{o}$ and $A_{o}$ Lamb Wave modes were possible by manipulating the incidence angle on the transmitter, both modes were centered at a $500 \mathrm{KHz}$ frequency. This method proved favorably to detect a hole - notch combination and notches with different orientations respectively. Figure 7 illustrates the amplitude of the waves scattered by the hole combined with reflections from notches of different sizes, versus receiver position. It should be observed that the location of the maximum value shifts to the right as the notch size increases. The figure also illustrates the receiver locations that were used to implement the location of the notch. In all cases, the points pertaining to the notch reflections were between $100 \mathrm{~mm}$ and $120 \mathrm{~mm}$.

As illustrated in

Figure 8 the results of processing the receiver signals acquired along the scanning line, are consistent with the actual location of the faults. However it could be appreciated that for plates with a hole-notch combination within a length of $1 / 8^{\prime \prime}-1 / 4^{\text {" }}$ an over prediction of the fault is experienced. The acquired signals were processed with the Hilbert-Huang transform to determine the signal energy as indicated in Section 3. After correcting for the time-delay associated with the trigger and wave arrival into the plate, the times of flight and the amplitudes of the scattering and reflected waves from hole and notch were obtained (Figure 5). The geometric approach as described in Section 5 was implemented for the times of flight corresponding to the range of 100 to $120 \mathrm{~mm}$, a region affected by the notch as illustrated in Figure 7. The results in

Figure 8 include a depiction of the actual shape of the hole- notch arrangement, angle, and horizontal notch. The overlap line indicates the points where the geometric methods determined a source of reflection. 


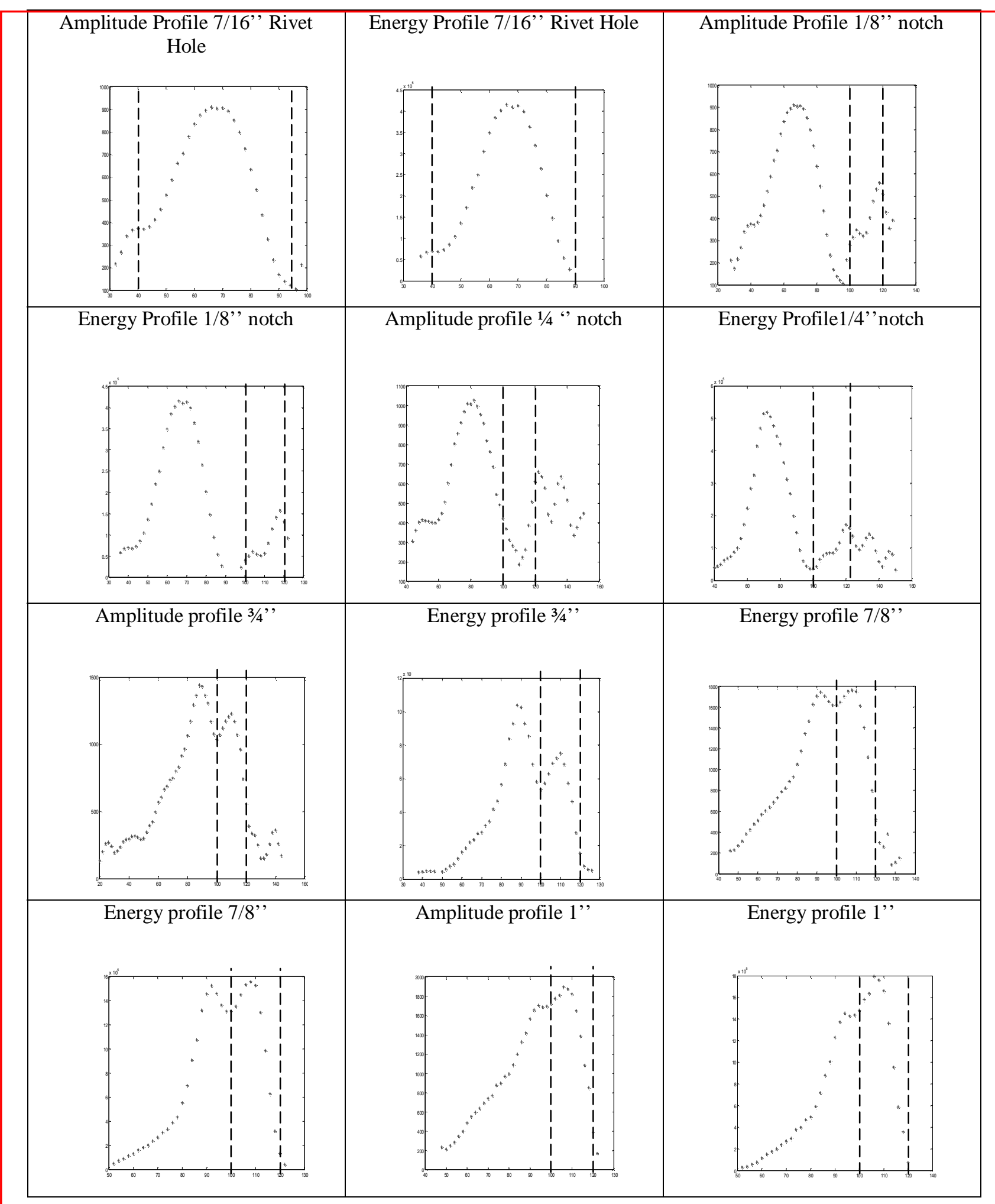

Figure 7 Amplitude of scattered and reflected wave energy form hole with a notch along line scan. 


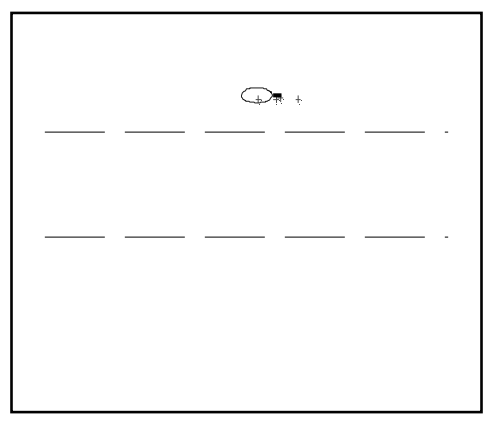

(a) $1 / 8$ " Notch Geometric Method II

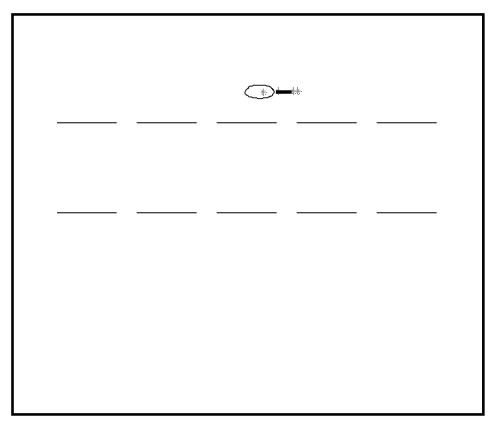

(d) $1 / 4$ " Notch Geometric Method I

(e) 5/8" Notch Geometric Method II

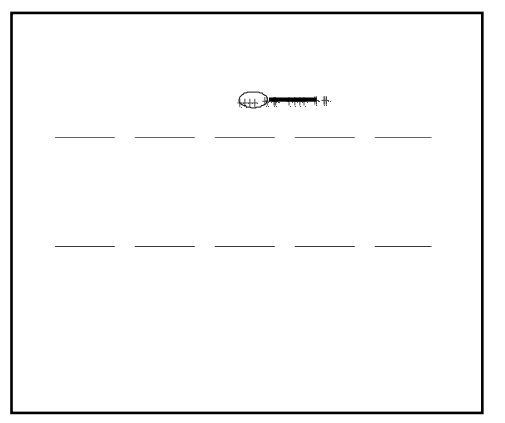

(g) 3/4' Notch Geometric Method II

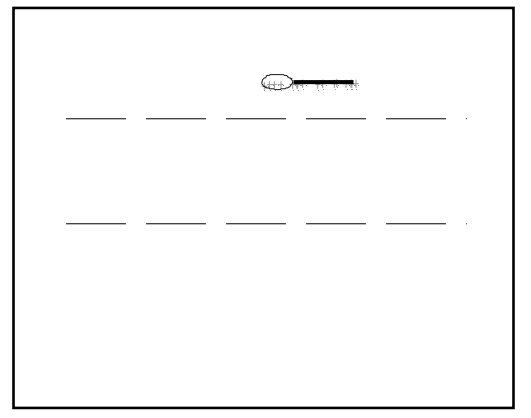

(m) $7 / 8$ " Notch Geometric Method I

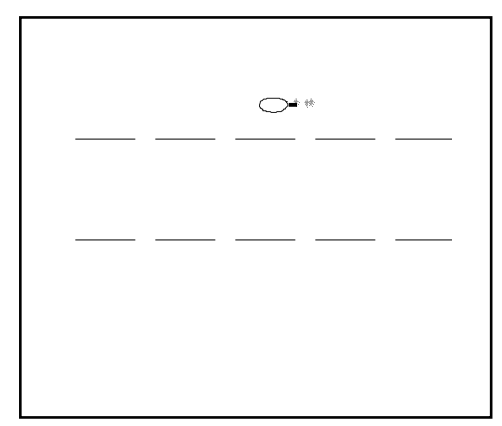

(b) $1 / 8$ "' Notch Geometric Method I
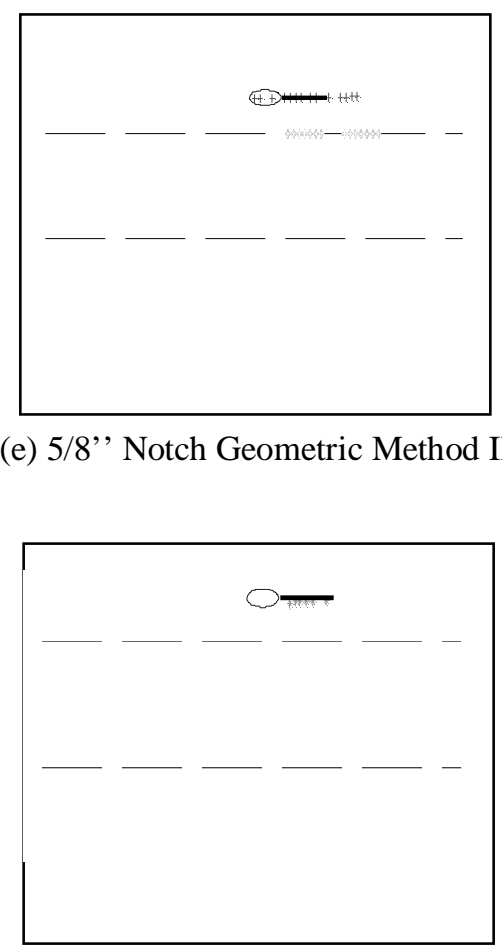

(h) 3/4” Notch Geometric Method I

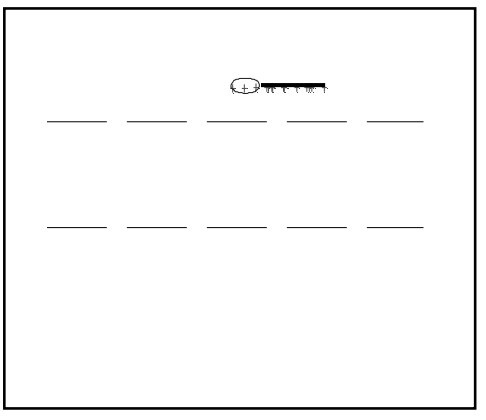

(n) 1" Notch Geometric Method II

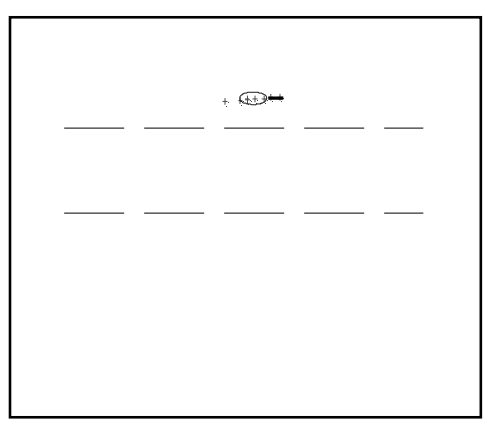

(c) 1/4" Notch Geometric Method II

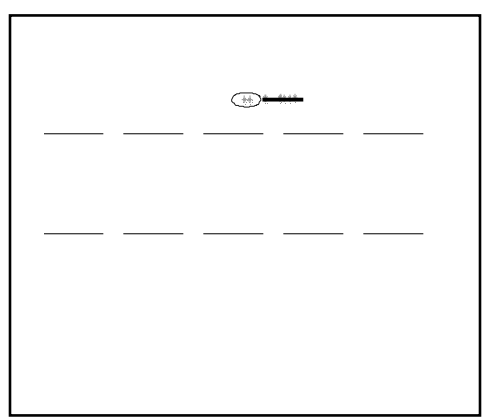

(f) 5/8' Notch Geometric Method I

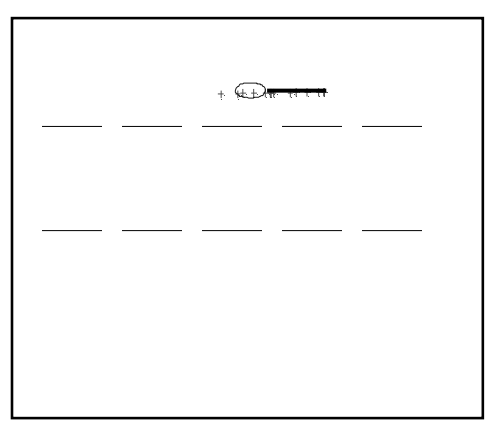

(1) 7/8" Notch Geometric Method II

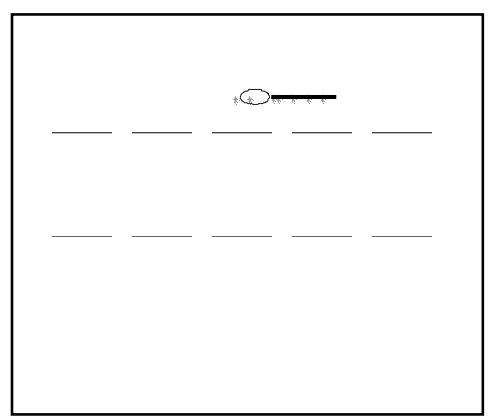

(o) 1" Notch Geometric Method I

Figure 8 Notch localization results, using the Geometric Method I and II. 

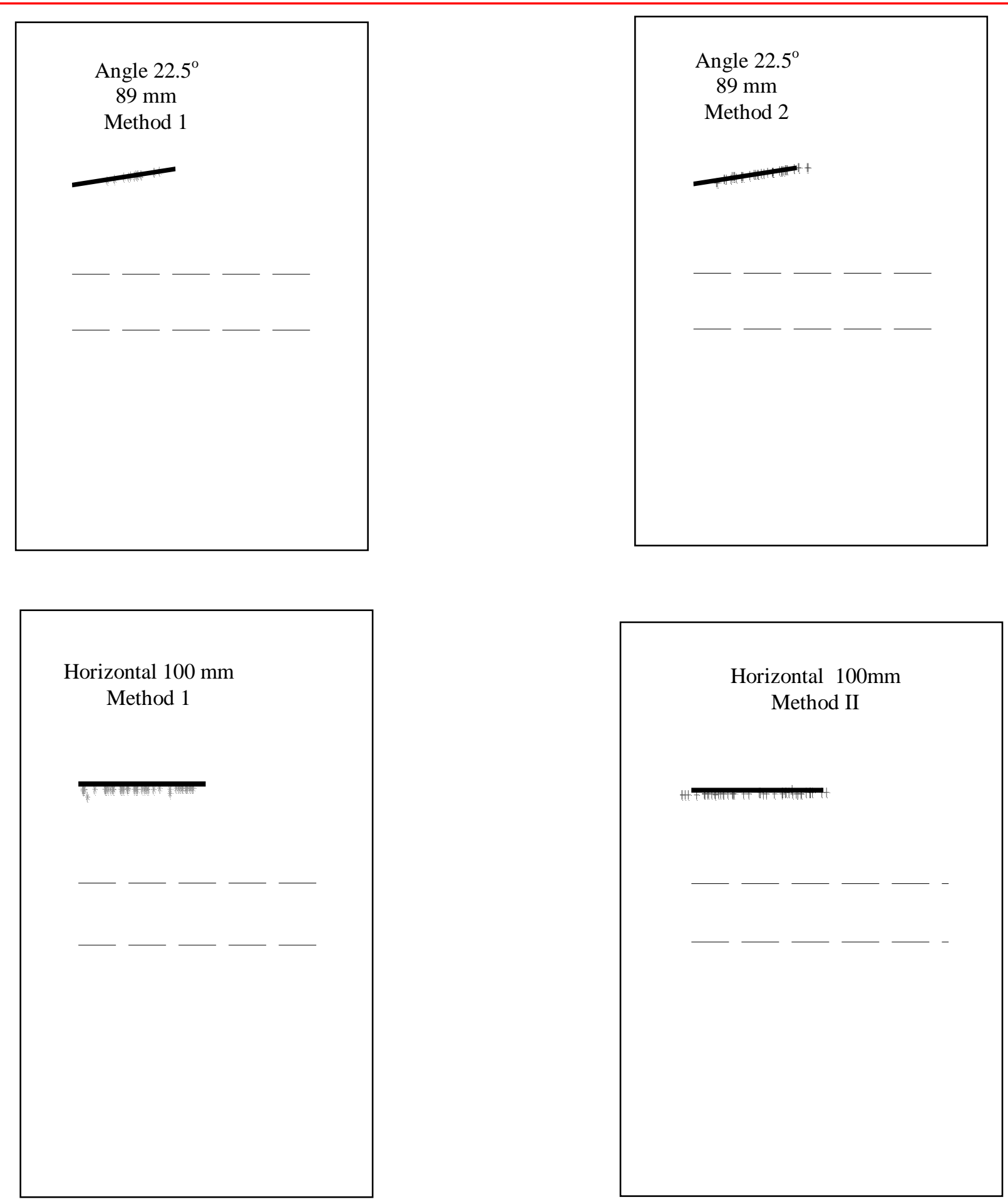

Figure 9. Localization results using Method I and II at an angle and horizontal. 


\section{CONCLUSION}

This paper has described a Lamb-wave scanning method that relies on the generation of $S_{0}$ and $A_{o}$ modes and the simultaneous movement of a transmitter-receiver arrangement, for the detection of notches at different orientations and simulating different scenarios. Two principal methods, Geometric Method I and Geometric Method II were utilized to determine coordinate points on a flaw line, by utilizing the arrival time information.

The results obtained and illustrated in Figure 9 confirm the improvement in signal energy as the transmitter moves in accordance to the receiver, thus demonstrating a reduction in signal attenuation and improvement in sensitivity. Another advantage attributed to this new approach, is the small length and time efficient scans that most be performed in order to acquire all necessary data.

In the case were a hole-notch arrangement was inspected amplitudes of the notch reflected energy outside a region affected by the back scattering from the hole was used to calculate coordinates of the source of the reflection. Line cans were conducted on various rectangular thin Aluminum plates with a center hole and various full-thickness notches ranging from $1 / 8$ "' to $1 / 4$ ''in long. The resulting coordinates outline the extent and relative direction of notches in these two different scenarios. One is having notches in a 0 to 22.5 degree orientation in respect to the far edge of the plate and the other is with notches of various sizes at a single rivet hole. Results of experiments conducted on 1.6mm-thick Aluminum rectangular plates, with an arrangement of notches as described compare favorably with the actual notches.

The following conclusions are stated on the proposed scanning approach:

- The location of the notch was always detected and correctly outlined.

- The extent of the flaw was accurately predicted of all the notches.

- The extent of the flaw was over-predicted of the shortest notch.

- The orientation of the predicted flaw was always in agreement with the actual notch orientations.

\section{ACKNOWLEDGEMENTS}

Efforts sponsored by the Air Force Office of Scientific Research, Air Force Material Command, USAF, under grant number F49620-00-1-0365. The US Government is authorized to reproduce and distribute reprints for governmental purposes notwithstanding any copyright notation thereon.

\section{REFERENCES}

1. P. Cawley, D. Alleyne, "The use of Lamb waves for the long range inspection of large structures," Ultrasonics 34, 1996, 287-290.

2. T. Kundu, K. Maslov, P. Karpur, T. E. Matikas, P.D. Nicolau, "A lamb wave scanning approach for the mapping of defects in [0/90] titanium composites," Ultrasonics 34, 1996, 43-49.

3. H.Kim, J.Song, D.Kwon"Measurement of phase velocity dispersion curves and group velocities in a plate using leaky Lamb waves," National Seminar ofISNT,2002,5-12

4. M.J.S. Lowe, D.N. Alleyne, and P. Cawley, "Defect detection in pipes using guided waves," Ultrasonics 36, 1998, 147-154.

5. T. Ghosh, T. Kundu and P. Karpur, "Efficient use of Lamb modes for detecting defects in large plates," Ultrasonics 36, 1998, 791-801

6. Z. Chang and A. Mal, "Scattering of Lamb waves form a rivet hole with edge cracks," Mechanics of Materials, 31, 1999, 197-204.

7. I.A. Viktorov, Raleigh and Lamb Waves - Physical Theory and Applications, Plenum Press, New York, 1967.

8. R. Osegueda, V. Kreinovich, S. Nazarian, and E. Roldan, "Detection of cracks at rivet holes in thin plates using Lamb-wave scanning," Paper 5047-06, in NDE for Health Monitoring and Diagnostics, SPIE, March 2003, 55-66.

9. F. DeVilla, E. Roldan, C. Tirado, R. Mares, S. Nazarian and R. Osegueda, "Defect Detection in thin plates using So Lamb wave scanning," in Proc. of NDE for Health Monitoring and Diagnostics, SPIE Vol 4335, Paper 21, March 2001.

10. N.E. Huang, Z. Shen, S.R. Long, S.R., M.C. Wu and Shih, H. H., et al., "The Empirical Mode Decomposition and Hilbert Spectrum for Nonlinear and Nonstationary Time Series Analysis,". Proc. Royal Society of London Series, A454, 1998, 903-995 